\section{Building Data Storage Systems And Data Networks To Support Digital Microscopy}

T.A. Dodson, T.A. Nolan, L.F. Allard, and E. Völkl Oak Ridge National Laboratory

As laboratories like the Materials Analysis User Center (MAUC) in the High Temperature Materials Laboratory, at Oak Ridge National Laboratory move from analog to digital imaging systems, the process for acquiring, processing, analyzing, and publishing research results is changing. In this case since original scientific data exist only in digital form, analog systems for gathering, storing, and transmitting data are being set aside in favor of digital systems. In order to adequately protect original scientific data and to ensure that digital laboratories have the same (or greater) functionality as analog laboratories, scientists must focus on building information systems that make data highly available, highly reliable, and quickly accessible. Meeting these three objectives imposes special requirements for both data storage systems and data networks

A data storage system for a digital microscopy laboratory must have a very large capacity (at MAUC, $30 \mathrm{~GB}$ ). In addition, the data stored within the system must be highly avallable and highly reliable. Highly available means that the data must be available both locally, and across the Internet, to any of the many different computer platforms that might be needed to process or analyze digital data. In our laboratory as in others, this includes Macintoshes, DOSWindows PC's, and UNIX workstations. Highly reliable means that the data must be safeguarded against tampering and secured for long periods of time. We have developed a simple architecture representing the data flows in our facility (Figure 1). Its shared storage system was developed to manage the data flows shown in the architecture and to answer the requirements of high reliability and availability.

The shared storage system incorporates components for storing data on-line and for archiving data off-line in a client/server arrangement that allows access via NFS from Macintoshes, DOSWindows PC's, Unix Workstations, and via FTP from authorized users on the Internet. To configure the on-line portion of this system, one must decide the amount of time that research data must be immediately accessible and work backwards to determine how much on-line storage is required. Figure 2 illustrates a sample calculation for our laboratory. CD-ROM'S serve as the off-line archive in the MAUC system. Writable CD-ROM's are used because of their long media life (30 years), relatively low cost ( $\$ 10 / \mathrm{blank}$ ), and wide readability (on Mac, PC, and Unix). Figure 3 shows how the pieces of our system fit together on the network. Operating this system requires great attention to detail. Regular backups are required to assure that scientific data is never lost. Timely archiving is required to prevent the data storage pool from becoming full and stopping further data acquisition. Security review and server access management are also required to avoid unauthorized access.

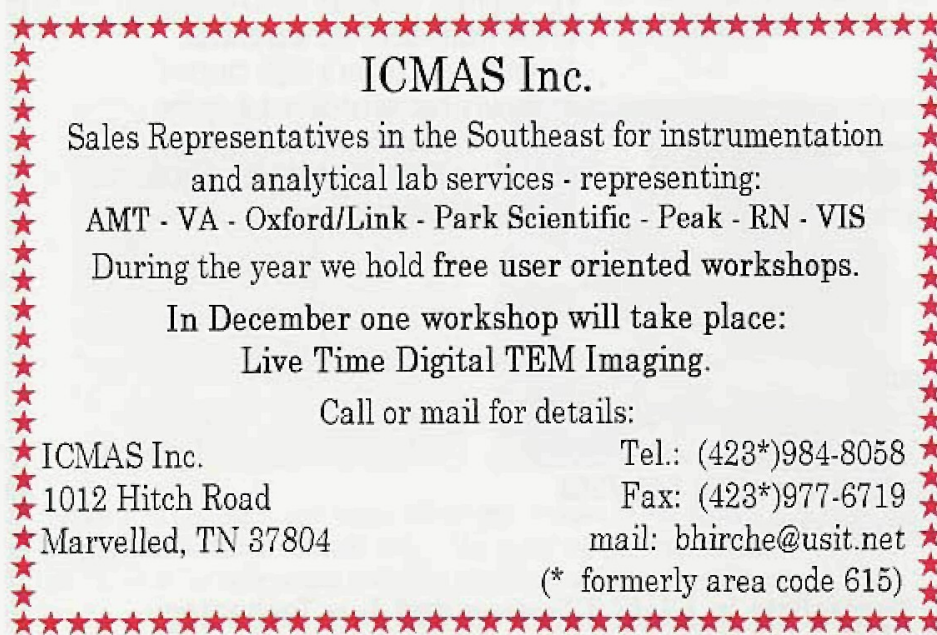

Since all the components of the information system used in the digital microscopy laboratory are interconnected via a network, the reliablity, availability, and speed of the network become limiting factors on the performance of the entire system. For networks, reliability refers to the quality of the data connection, availability refers to the amount of time that the network is up and useable, and the speed refers to the amount of information that the network is capable of transmitting per unit time. A range of network technologies exist that deliver different levels of reliability and speed. Most laboratories begin with Ethernet (10 Mbps, shared) and move to faster systems as they see performance bottlenecks. Bottlenecks arise soon after the conversion to digital begins. Users transmitting 2 MB images soon appreciate speeds (100 Mbps and greater) that were previously reserved for connecting large campus networks.

When evaluating proposed network upgrades, consider how fast each network operates and whether this bandwidth is guaranteed to each port (switched) or shared between all the users on the network. In general, the higher the bandwidth guaranteed to each port, the better. We currently use a combination of Ethernet (10 Mbps, shared), switched Ethernet (10 Mbps, dedicated), and FDDI (100 Mbps, shared) interconnected as shown in Figure 4. As our bandwidth requirements grow, it is anticipated that switched FDDI and ultimately Asynchronous Transfer Mode (ATM, 155 Mbps, dedicated) will be installed to the desktop.

Although the systems described above are adequate for the data types handled today (images, spectra, and reports), digital microscopy is rapidly evolving into remote microscopy. As systems for TelePresence Microscopy constructed as part of an Electronic Laboratory (E-lab) mature, bi-directional streams of image data and audio will further increase the requirements for both networks and storage systems. ${ }^{1}$ It is impossible to accurately estimate performance requirements for the Electronic Laboratory. Certainly laboratories that have gradually improved their data storage systems and data networks will be closest to operating an Electronic Laboratory. However, improving the speed of data network facilities and the capacity/structure of data storage systems can require significant funds and long lead times. Microscopists should begin communicating the vision of these new systems to managers and support staff while planning to meet new requirements today.

1. N.J. Zaluzec et al., LabSpace/TPM-A National Electronic Laboratory Infrastructure initiative, Argonne National Laboratory (1994).
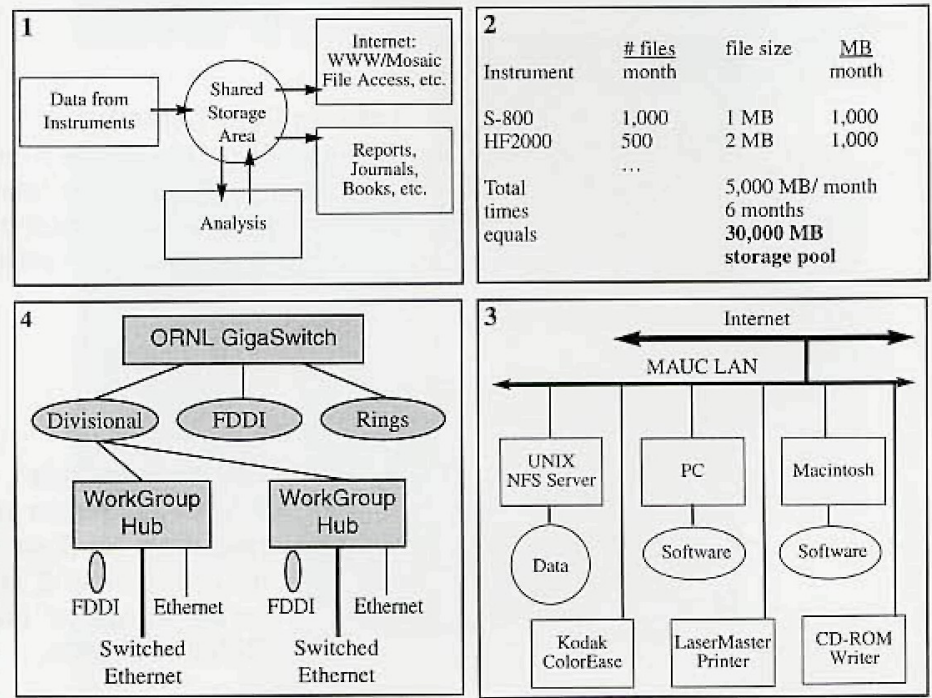

Figure 1: Overview of information flows handled by the MAUC storage architecture

Figure 2: Sample calculation for determining the size of on-line storage requirements for the MSUC system.

Figure 3: Diagram of the implementation of the MAUC storage architecture for digital microscopy data.

Figure 4: Diagram showing the current structure of MAUC data metwork

Reprinted from Proceedings, Microscopy and Microanalysis 1995, with the premission of the Microscopy Society of America. 


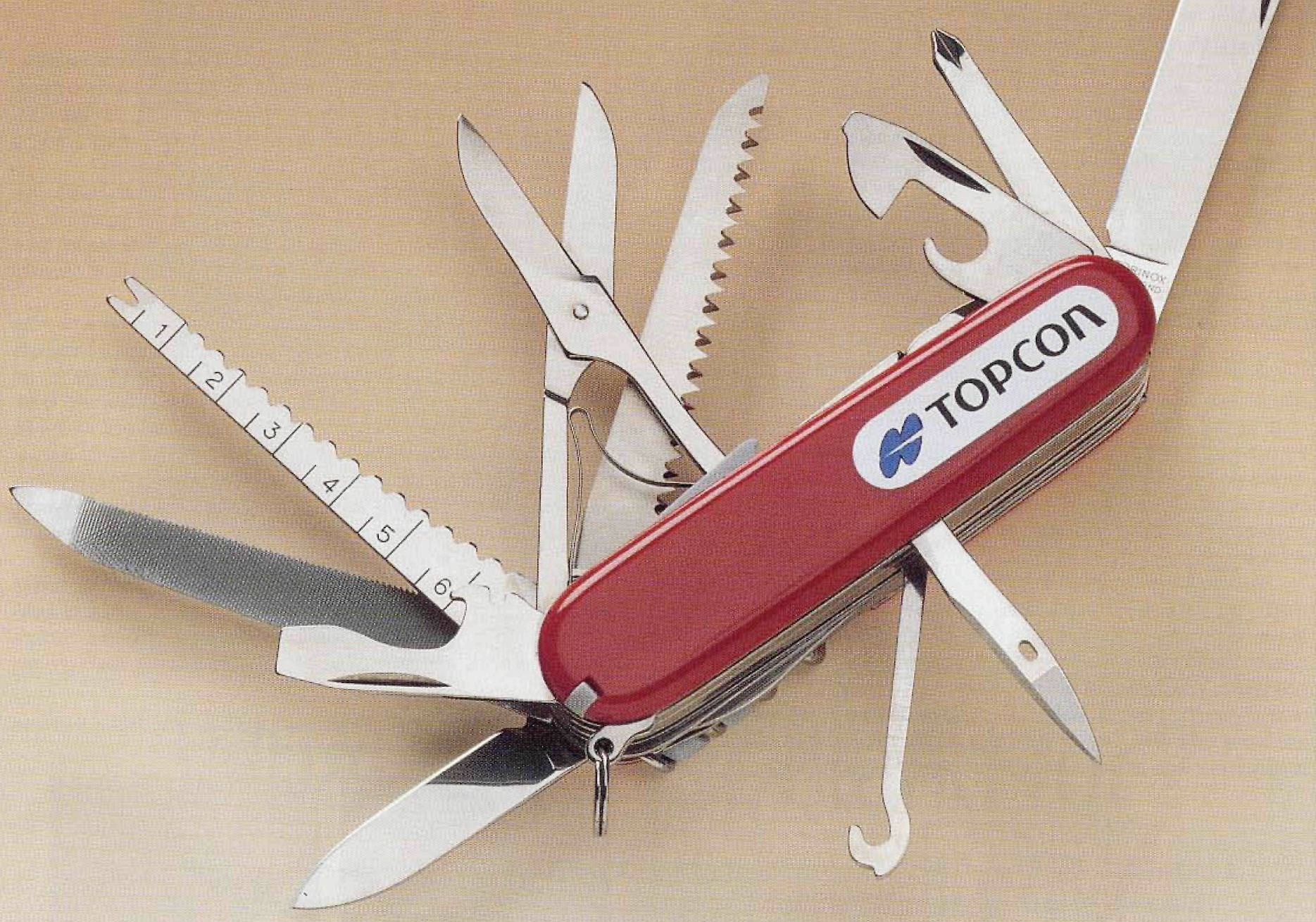

\section{UNEQUALED VERSATILITY}

Topcon Scanning Electron Mieroscopes in a Unique Class

You value the versatility of a Swiss Army Knife. Now, you can have that same kind of versatility with Topcon's 500 Series SEMs for your scanning electron microscopy tasks. The versatility to meet your needs today and tomorrow.

\section{UItimate SEM Capabilities}

Choose from a complete family of SEMs Tungsten (W), $\mathrm{LaB}_{6}$, and Field Emission.

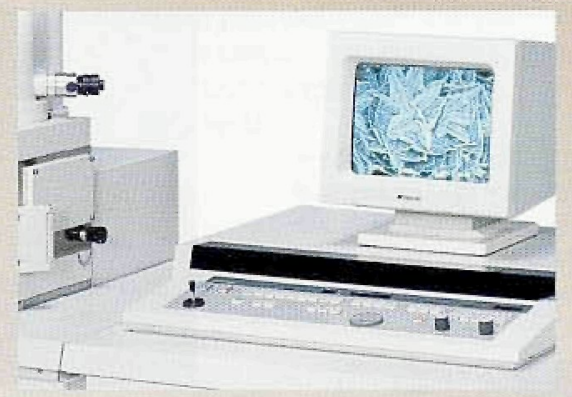

All have windows mouse-based computer controls or traditional automated controls, or both, to provide access to all SEM func- tions. Comprehensive digital imaging and archiving provide full image manipulation. Unique WET-SEM capability gives you the real image quickly and easily. Compare and you'll agree that no other SEM comes close in versatility, ease of use, reliability and value. It's unequaled. But see for yourself. Call us at 1-800-538-6850 or write to TOPCON, 37 West Century Road, Paramus,

New Jersey 07652 .
TOPCON

TOPCON TECHNOLOCIES INCORPORATED 